\title{
Accomplishing breakthroughs in behavioural medicine research
}

\author{
Karina W. Davidson, Simon L. Bacon, Gary G. Bennett, Elizabeth Brondolo, Susan M. Czajkowski, \\ Michael A. Diefenbach, Elissa S. Epel, Karen Matthews, Tracey A. Revenson, John Manuel Ruiz, \\ Suzanne C. Segerstrom and Behavioral Medicine Research Council
}

$\mathrm{T}$ o the Editor - Research progress for behavioural medicine has been observably fragmented and incremental, and it often occurs by accident rather than design. The Behavioral Medicine Research Council aims to lead coordinated efforts to identify target priorities for the science proposed, conducted, and implemented by the field of behavioural medicine.

Globally, behavioural risks are associated with the largest decrement in disability-adjusted life-years ${ }^{1}$. In the United States, an analysis of the 2016 Global Burden of Disease study results revealed that $19.5 \%$ of total disability-adjusted life-years could be attributed to behavioural risk factors ${ }^{2}$. Consequently, many governmental agencies, health policy entities, and funding agencies now focus on behaviour as a way to improve the health of their nations' people.

Behavioural medicine is the interdisciplinary field of science focused on developing and integrating behavioural, psychosocial, and biomedical science knowledge and methods to better understand health and illness. The field aspires to improve primarily through tertiary prevention, diagnosis, and treatment of all diseases and health states affected by behaviours ${ }^{3}$. However, research progress for behavioural medicine has been fragmented and frequently happens by accident rather than design. The field's four primary professional societies (the Academy of Behavioral Medicine, the American Psychosomatic Society, the Society of Behavioral Medicine Research, and the Society for Health Psychology) recognized the need to accomplish breakthroughs in the area of behavioural medicine and build on our accomplishments to achieve a more cumulative, rather than fragmented, science. In December 2018, the Behavioral Medicine Research Council (BMRC) was created to disrupt the scientific culture of the behavioural medicine research community ${ }^{4}$, address the field's fragmentation, and lead coordinated efforts to identify target priorities for transformative science needed to advance behavioural medicine ${ }^{5}$.

\section{The problem}

Solutions to contemporary health challenges require an orchestrated plan of discovery, intervention development, optimization, and scaling/implementation. Tackling the complex behavioural issues involved in managing the COVID-19 pandemic is just one of many such challenges. The BMRC is developing key strategies about the processes that should be considered with behaviour-based research in response to an acute crisis. However, designing immense strategic, complex, or discovery-based behavioural medicine research is, for many researchers, not perceived as a current possibility. Reinforcements for this type of behavioural medicine research do not exist, nor do the resources to enact it.

A minimum of two levels are integral in considering how incentives shape scientific communities to focus on simple rather than complex approaches ${ }^{6}$. First is the process by which individual scientists make choices about the focus and level of complexity of their work. Second are selective processes that govern new scientists' training and the formation of manageable research groups, areas, and research capacities ${ }^{6}$. The BMRC was created to tackle both of these issues and focus on select target priorities.

Individual efforts and happenstance currently drive most of the accomplished progress in the field of behavioural medicine. As academics, researchers, and scientists, we propose projects that have the best chance of receiving funding; in evaluating proposals worth funding, scholars tend to select (often without making a prior decision to do so) conformist science rated as having the highest funding merit ${ }^{6}$. We focus exclusively on testing scientific ideas that can be accomplished in just a few years, as most federally funded grants are slated for 5 years (or fewer) of funding. Furthermore, we complete science in areas in which only a few expertise domains are needed so as to avoid excessive complexity. Given that the field was established 40 years ago ${ }^{3}$, perhaps the time has come for a revolution in the manner in which we identify, prioritize, and advance behavioural medicine research ${ }^{7}$.

\section{The proposed solution: learn from the best}

We propose reviewing best practices from other fields and determining which may offer new ways for behavioural medicine to advance more rapidly with an increased focus on the larger complex problems the field needs to address.

Set up the right structure. Children's Oncology Group is the world's largest organization devoted exclusively to childhood and adolescent cancer research. Its structure began with four competing pediatric groups that merged in 2000 (ref. ${ }^{8}$ ). The Children's Oncology Group has nearly 100 active clinical trials open at any given time ${ }^{9}$ (they received more than US $\$ 146,000,000$ in 2019). It offers care and clinical trial enrollment for more than $90 \%$ of the 14,000 children and adolescents diagnosed with cancer each year in the United States. Imagine a world in which any person with health behaviour problems was enrolled in a trial and provided care based on the most recent scientific advances. Though establishing a single entity of clinical trial infrastructure for all behavioural medicine trials seems unimaginable, others have accomplished it for the sake of moving the science and its clinical applications forward.

Currently, the field of behavioural medicine has isolated groups of investigators who have sporadically conducted multi-site trials. However, several actions are needed to accomplish such a network for behavioural medicine trials. The International Behavioural Trials Network (https:// www.ibtnetwork.org/) was established in 2013 to facilitate global improvement in methodological quality of behavioural trials, a critical first action. Next, the trials needed should be identified, prioritized, and conducted-actions the BMRC is charged to fulfill. However, the barrier of securing long-term, stable funding, as in the pediatric oncology field, must also be overcome.

\section{Use the right process}

The United States Department of Defense's Defense Advanced Research Projects Agency 
(DARPA) has produced an unprecedented number of breakthroughs. Arguably, it has the longest-standing, most consistent program of radical inventions in history ${ }^{10}$. With its unconventional approach, speed, and effectiveness, DARPA has created a "special forces" process of innovation. Reflections from past leaders suggest that this is an instantiation of the 'use-inspired basic research' section of Pasteur's Quadrant ${ }^{11}$. As described by others, it entails pushing the frontiers of basic science to solve a well-defined, use-inspired need. This innovative approach is the type of process that the BMRC could adopt to focus behavioural medicine on the selected priorities.

The DARPA process consists of three parts and one overarching culture. First, it sets an ambitious goal that tackles the most critical problems and thus advances science. Second, for a time-limited period, it assembles the best minds from industry and academia in creating diverse, agile, and scalable teams in one specific science area that can select and execute science in a way that deviates substantially from mainstream processes. Third, it 'flips the script': instead of starving scientists for resources so that large amounts of time are required for seeking funding, it instead starves scientists of time and provides unlimited resources. Its culture empowers scientists and the DARPA project managers to take risks on innovative ideas, expect failures, experience autonomy, and contribute to an environment of excitement and trust. Other innovators, such as the Gates Foundation, use similar processes and create a similar culture. They issue challenges such as the Healthy Longevity Global Competition ${ }^{12}$ that ask for the most crucial goal to be attained and then provide extraordinary resources to the teams who offer unique and exciting proposals to accomplish it. The overall function of these programs of science would not be recognizable to most scientists currently conducting research in behavioural medicine; we are rarely, if ever, asked, "if you had unlimited resources and a tight timeframe, what single behavioural medicine problem would you solve now?" and then directed to "propose your solution and execute it." Though DARPA's and the Gates Foundation's processes are unique to their respective endeavours, their general approaches to pursuing and accomplishing progress are noteworthy. Imagine if the entire behavioural medicine field was tasked with solving structural racism with regard to healthcare, climate change, or COVID-19 vaccine hesitancy and then provided the necessary funds to test their proposed scientific hypotheses immediately without penalizing initial failure. What could the field achieve?
Incentivize the right behaviour. Creating incentives for long-term capacity-building and science system change is particularly difficult. We need a critical mass of national and international pioneering institutions that embrace the principles of transformative science as part of their strategic mission, and these have traditionally been nonprofit foundations. MacArthur Genius awards-created to serve long-term research capacity-building to address neglected or absent science areas-are one way that creative, risky, and innovative advances are awarded and reinforced for individual scientists. The National Cancer Institute of the National Institutes of Health and other entities such as Cancer Research U.K. have experimented with 'sandpits' as a way to incentivize the proper behaviour at the individual-scientist level. Sandpits are creativity competitions among randomly formed teams of scientists wherein the best idea is funded for an investment of only about 5 days. Partnering with foundations and funding agencies to run these sandpits regularly and on a focused topic would allow the BMRC to enact its mission and is therefore one of the committee's initial actions.

\section{Moving forward}

These are daunting challenges and ambitious undertakings to alter the course of an area such as behavioural medicine. Building on the vision and initial plans for the $\mathrm{BMRC}^{4}$, the organization will focus on:

- Drawing from the successful efforts of the best organizations and agencies to create processes that advance science rapidly

- Establishing transparent and public processes for rapid topic identification and prioritization

- Conducting comprehensive evidence synthesis and identifying critical research gaps needed in discovery-based, clinical, and implementation behavioural science

- Locating funding partners

- Providing the intellectual infrastructure to permit researchers to advance towards solutions as quickly as possible

- Ensuring inclusivity in every part of the initiative

- Building a pipeline of researchers with the interpersonal, strategic, and creative skills necessary for collaboration and innovation

We are in an exciting age for the science of behavioural medicine research. Our scientific community is in a prime position to creatively disrupt the scientific methods and approaches employed in the field.
More than ever, researchers who recognize the limitations of individual research groups and single methods are connecting across disciplines and countries and sharing resources through Open Science practices $^{13,14}$. Designing structures, processes, and incentives to transform our field and its science can be done explicitly, and the BMRC will do so. Uniting these and other initiatives will cultivate a more cumulative science of behavioural medicine that can inform policy, practice, and scientific progress.

Karina W. Davidson (D1凶, Simon L. Bacon (D)2, Gary G. Bennett ${ }^{3}$, Elizabeth Brondolo ${ }^{4}$,

Susan M. Czajkowski ${ }^{5}$, Michael A. Diefenbach', Elissa S. Epel ${ }^{6}$, Karen Matthews ${ }^{7}$,

Tracey A. Revenson ${ }^{8}$, John Manuel Ruiz ${ }^{9}$, Suzanne C. Segerstrom ${ }^{10}$ and Behavioral Medicine Research Council ${ }^{\star}$

${ }^{1}$ Feinstein Institutes for Medical Research, Northwell Health, Manhasset, NY, USA. ${ }^{2}$ Department of Health, Kinesiology \& Applied Physiology, Concordia University, Montreal, Quebec, Canada. ${ }^{3}$ Trinity College of Arts \& Sciences, Duke University, Durham, NC, USA. ${ }^{4}$ College of Liberal Arts and Sciences, St. John's University, New York, NY, USA. ${ }^{5}$ Health Behaviors Research Branch, Behavioral Research Program, Division of Cancer Control and Population Sciences, National Cancer Institute, Bethesda, MD, USA. ${ }^{6}$ Weill Institute for Neurosciences, School of Medicine, University of California, San Francisco, CA, USA. ${ }^{7}$ Department of Psychiatry, University of Pittsburgh, Pittsburgh, PA, USA. ${ }^{8}$ Department of Psychology, Hunter College, New York, NY, USA. ${ }^{9}$ College of Science, University of Arizona, Tucson, AZ, USA. ${ }^{10}$ College of Arts \& Sciences, University of Kentucky, Lexington, KY, USA. ${ }^{*} A$ list of authors appears at the end of the paper.

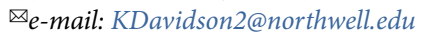

Published online: 14 June 2021

https://doi.org/10.1038/s41562-021-01134-4

References

1. Gakidou, E. et al. Global, regional, and national comparative risk assessment of 84 behavioural, environmental and occupational, and metabolic risks or clusters of risks, 1990-2016: a systematic analysis for the Global Burden of Disease Study 2016. Lancet 390, 1345-1422 (2017).

2. U. S. Burden of Disease Collaborators. et al. The State of U.S. Health, 1990-2016: burden of diseases, injuries, and risk factors among U.S. states. JAMA 319, 1444-1472 (2018).

3. Ruiz, J. M. \& France, C. R. Introduction to the special series-behavioral medicine at 40: origins, achievements, and future directions. J. Behav. Med. 42, 1-4 (2019).

4. Freedland, K. E. The Behavioral Medicine Research Council: its origins, mission, and methods. Health Psychol. 38, 277-289 (2019).

5. Diefenbach, M. A., Phillips, A. \& Bullock, L. Provocative questions in behavioral medicine and the acceleration of our science. Ann. Behav. Med. 54, 920-923 (2020).

6. O'Connor, C. The natural selection of conservative science. Stud. Hist. Philos. Sci. 76, 24-29 (2019).

7. Freedland, K. E. The evolution of professional societies in behavioral medicine. J. Behav. Med. 42, 52-56 (2019).

8. O'Leary, M., Krailo, M., Anderson, J. R. \& Reaman, G. H. Progress in childhood cancer: 50 years of research collaboration, a report from the Children's Oncology Group. Semin. Oncol. 35, 484-493 (2008). 
9. Children's Oncology Group. About us. Children's Oncology Group https://childrensoncologygroup.org/index.php/aboutus (2020).

10. Dugan, R. E. \& Gabriel, K. J. "Special Forces" innovation: how DARPA attacks problems. Harv. Bus. Rev. October, 74-84 (2013).

11. Stokes, D. E. Pasteur's Quadrant: Basic Science and Technological Innovation. (Brookings Institution Press, 1997).

12. National Academy of Medicine. U.S. National Academy of Medicine Healthy Longevity Global Grand Challenge. https://healthylongevitychallenge.org/ (National Academy of Medicine, 2020).

13. Good Christopherson, E. Rita Allen Foundation Stories. Rita Allen Foundation (2020); http://ritaallen.org/stories/ announcing-the-2019-rita-allen-foundation-scholars

14. Nosek, B. A. et al. Promoting an open research culture. Science 348, 1422-1425 (2015)

Acknowledgements

This work was supported by the National Cancer Institute (R13CA228496) and the National Library of Medicine
(R01LM012836) of the National Institutes of Health in addition to the professional organizations of the Academy of Behavioral Medicine Research, the American Psychosomatic Society, the Society for Health Psychology, and the Society of Behavioral Medicine. No sources of funding had any role in this opinion piece. We thank A. Dominello and S. D'Angelo for their contributions to this manuscript.

Author contributions

All authors except K.W.D. are listed alphabetically.

Competing interests

The views expressed in this paper are those of the authors and do not represent the views of the National Institutes of Health, the United States Department of Health and Human Services, or any other government entity. K.W.D. is a member of the United States Preventive Services Task Force (USPSTF). This article does not represent the views and policies of the USPSTF. The authors report no financial or personal relationships with other people or organizations that could inappropriately influence their work in this manuscript.

Behavioral Medicine Research Council

Simon L. Bacon ${ }^{2}$, Gary G. Bennett ${ }^{3}$, Elizabeth Brondolo ${ }^{4}$, Susan M. Czajkowski ${ }^{5}$, Karina W. Davidson', Michael A. Diefenbach', Elissa S. Epel ${ }^{6}$, Karen Matthews ${ }^{7}$,

Tracey A. Revenson ${ }^{8}$, John Manuel Ruiz ${ }^{9}$ and Suzanne C. Segerstrom ${ }^{10}$ 